\title{
Multiple measures of refractive state of a test eye making use of a Nidek ARK-10000 OPD-
}

\author{
sca n
}

\section{WDH Gillan*}

Department of Optometry, Optometric Science Research Group, University of Johannesburg, PO Box 524, Auckland Park, 2006 South Africa

<wdhg@na.rau.ac.za >

\begin{abstract}
Introduction: It is important for the users of automated instruments that measure refractive state to know that the results obtained from such instruments are accurate and repeatable. This study was conducted to investigate the repeatability of multiple measures of refractive state using a Nidek ARK-10000 OPD-scan.
\end{abstract}

Method: 150 measurements of refractive state of a test eye were obtained using an OPDscan. The method of focusing and triggering the instrument was varied for three sets of 50 measurements.

Results: The results of this study suggest that the OPD-scan gives reliable and accurate measures of refractive state when taking multiple measurements. Although the means of the various sets of measurements were significantly different, clinically the differences are probably insignificant.

\section{Introduction}

The Nidek ARK-10000 OPD-scan is an instrument that is designed to provide information relating to autorefraction, autokeratometry, corneal topography, wavefront analysis and pupillometry ${ }^{1,2}$. The OPD scan can be used in one of two modes. Autorefraction and autokeratometry are measured in one mode and wave- front information and topography are determined in the second mode. It is important that automated instruments are reliable and accurate for both clinicians and researchers. Many reports have been published that have evaluated the performance of autorefractors, both as individual instruments and as comparisons between instruments $^{3-9}$. In general, autorefractors are considered to provide reasonably accurate and repeatable measurements of refractive state. Other studies comparing autorefractors, conventional refraction and retinoscopy have found that autorefractors give an accurate indication of the refractive state of the eye $\mathrm{e}^{12-15}$.

Many researchers take multiple (50 or more) measurements of refractive state during their research projects ${ }^{16-20}$. For this reason one needs to be confident that an instrument provides accurate and repeatable measures of refractive state. Studies ${ }^{21,22}$ investigating the repeatability of autorefractor measurements have shown that automated measurements of refractive state are repeatable. Bullimore, Fusaro and Adams ${ }^{22}$ have gone so far as to state: "automated refraction is more repeatable than subjective refraction and therefore more appropriate for studies of myopia progression".

Gillan $^{23}$ has shown that the length of time that an instrument is left on plays a role in

*DipOptom DPhil(RAU) CAS(NewEnCO) FAAO FIACLE

Received 20 April 2006; revised version accepted 8 December 2006 
the repeatability of multiple measurements of refractive state. It has also been shown that the way an autorefractor is focused and whether the instrument is refocused after every measurement influences the variation that occurs in measures of refractive state ${ }^{24}$.

A test eye is considered to be static and incapable of accommodative or other fluctuations. The test eye therefore makes a useful object to investigate the accuracy and repeatability of multiple measurements of refractive state. In all probability any changes that are determined while taking measures of refractive state of a test eye are due to operator or instrument error or fluctuation. The aim of this study is to investigate the repeatability of multiple measures of refractive state taken using an OPD-scan and at the same time to determine if the method of triggering the instrument affects its accuracy.

\section{Method}

The Nidek ARK-10000-OPD scan has various modes for measuring refractive state of an eye in the autorefractor mode: firstly the instrument can be set so that the user has to depress the trigger button when the user believes that the instrument is properly focused and secondly the instrument can automatically trigger itself when properly focused. This study involves using three different methods of focusing and triggering the instrument. Fifty measurements of refractive state of a Nidek test eye were taken having the researcher focus the instrument and deciding when to depress the trigger button. The instrument was refocused after each individual measurement. A print-out was made after each set of ten measurements. A second set of fifty measurements were taken of the test eye where the instrument was set to trigger itself automatically when focused. The instrument was then moved back and realigned before the instrument triggered itself once again. A printout was made after each set of ten measurements. Another fifty measurements were collected where the instrument triggered itself when focused and ten measures
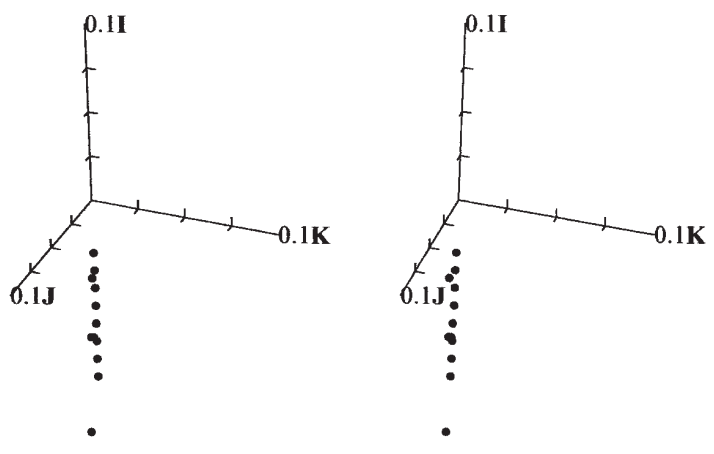

Figure 1a. Scatter plot of 50 measurements of refractive state of a test eye. Origin of axes is set at $-5 \mathrm{D}$ for this and all subsequent figures. Operator refocused the instrument after each measurement and decided when to trigger the measurement. Length of axes: $0.1 \mathrm{D}$. For details see text.
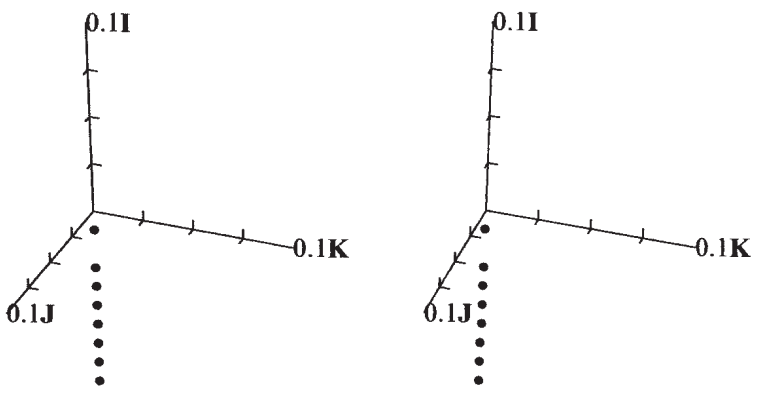

Figure 1b. Scatter plot of 50 measurements of refractive state of a test eye. Operator refocused the instrument after each measurement and the instrument triggered itself when adequately focused. Length of axes: $0.1 \mathrm{D}$. For details see text.
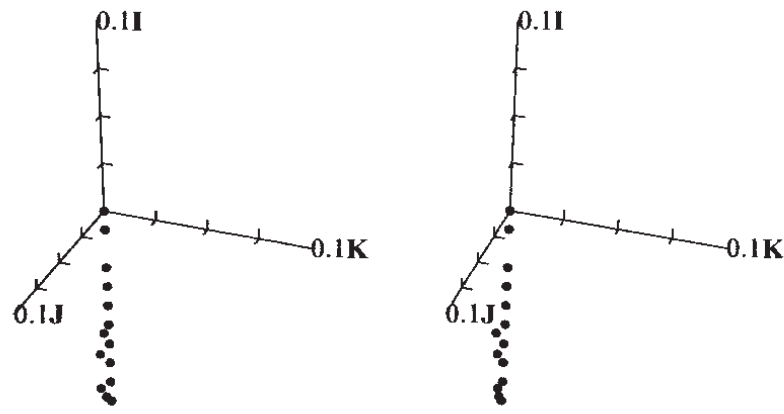

Figure 1c. Scatter plot of 50 measurements of refractive state of a test eye. Operator focused the instrument once, ten measurements were taken at a time with no refocusing of the instrument. The instrument triggered itself when adequately focused. Length of axes: $0.1 \mathrm{D}$. For details see text. 
of refractive state were taken at a time without refocusing the instrument. A total of 150 measurements of refractive state were taken of the test eye. The instrument was set to measure refractive state in $0.01 \mathrm{D}$ steps with the vertex distance set at $12 \mathrm{~mm}$ (the calibration vertex distance of the test eye). The manufacturer's stated dioptric power of the test eye was $-4.92 \mathrm{D}$ at a $12 \mathrm{~mm}$ vertex distance. The data were analyzed using methods developed by Harris and software developed by Harris and Malan and modified by Rubin.

\section{Results}

Figure 1 shows scatter plots representing data collected making use of different methods of activating the OPD-scan. The origin of the axes in all scatter plots in this article is $-5.00 \mathrm{D}$. Axis length depends on the data collected and will be indicated in the text. Figure 1a shows 50 measurements of refractive state collected by refocusing the OPD scan after each measurement with the operator deciding when to trigger the measurement. Axis length in Figure 1a is $0.1 \mathrm{D}$. An elongated cluster of data points is seen with most of the points in a straight line indicating mostly stigmatic (spherical) variation of the measurements. The extent of the stigmatic variation is approximately $0.1 \mathrm{D}$. Also seen are several data points that are slightly displaced away from the seemingly straight line of data points. The displaced points show measurements of refractive state that include antistigmatic (astigmatism) components of refractive state. The antistigmatic components of refractive state are small. The mean of the 50 measurements is shown in Table 1 in conventional as well as component notation.

Figure $1 \mathrm{~b}$ shows 50 measurements taken by refocusing the instrument after each measurement with the instrument set to trigger itself. The axis length is $0.1 \mathrm{D}$. An elongated cluster of points is seen indicating stigmatic variation in the measurements. There are no points indicating antistigmatic variation. The extent of the cluster is approximately $0.075 \mathrm{D}$. The mean of the 50 measurements is seen in Table 1.

Figure $1 \mathrm{c}$ represents 50 measurements obtained by initially focusing the instrument and having the instrument trigger itself when adequately focused. The instrument was not refocused after each measurement. Ten measurements were taken at a time and then printed. The axis length is 0.1 D. The stigmatic extent of the elongated cluster is approximately $0.1 \mathrm{D}$. A number of data points show antistigmatic components of refractive state. The mean of the 50 measurements can be seen in Table 1. A multivariate analysis of variance (MANOVA) was conducted on the data shown above. At the $95 \%$ level of confidence all means and variances were shown to be significantly different (even though the differences are small).

The presentation in Figure $1 \mathrm{~b}$ is unusual as

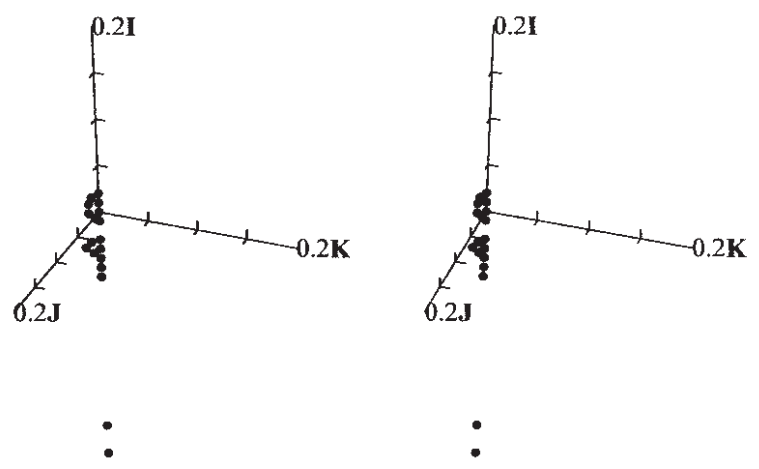

Figure 2. Scatter plot of 50 measurements of refractive state of a test eye taken two months after initial measurements. Operator refocused the instrument after each measurement and the instrument triggered itself when adequately focused. Length of axes: 0.2 D. For details see text.
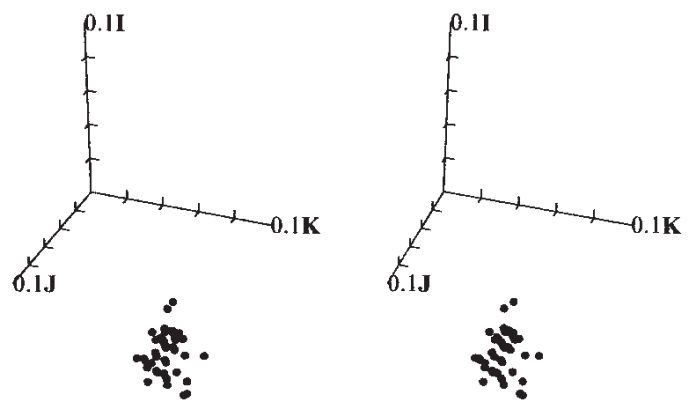

Figure 3. Scatter plot of 50 measurements of refractive state of a test eye obtained using a Nidek ARK-700 autorefractor. An increased amount of antistigmatic variation is seen. Operator refocused the instrument after each measurement and decided when to trigger the measurement. Length of axes: 0.1 D. For details see text. 
there are no antistigmatic measures of refractive state. All other investigations that have been conducted by members of the Optometric Science Research Group (OSRG) making use of multiple measurements obtained using autorefractors have shown the presence of antistigmatic components of refractive state. It was decided to take another 50 measures of refractive state using the same methods as those used to collect the data for Figure 1b. The measurements were taken approximately two months later. Figure 2 shows the 50 measure-
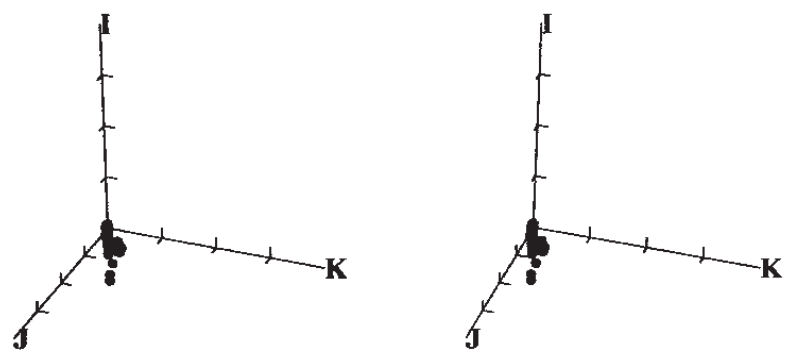

Figure 4a. Two hundred measurements of refractive state of a test eye are shown on one set of axes. Axis length: $1 \mathrm{D}$.
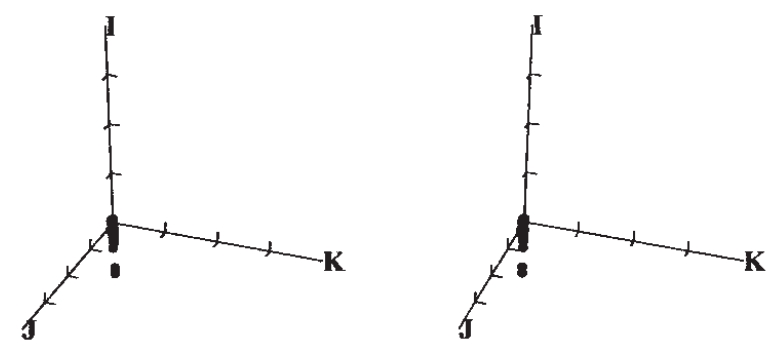

Figure $\mathbf{4 b}$. The same data as that seen in Figure $4 \mathrm{a}$ is represented here. The 50 measurements of refractive state obtained using the ARK-700 autorefractor are excluded. A tight cluster is seen with little antistigmatic variation. Two possible outliers are seen positioned below (in this orientation) the primary cluster.

ments collected at the later date. The axis length is $0.2 \mathrm{D}$. A number of interesting phenomena can be seen. The stigmatic elongation of the data is evident, however, two data points positioned at the bottom of the scatter plot (in this orientation) could possibly indicate outliers. The stigmatic extent of the cluster, including the two possible outliers, is $0.15 \mathrm{D}$.

\begin{tabular}{|l|l|l|l|l|l|l|}
\hline \multicolumn{6}{|l|}{ Table 1. Means of 50 measurements are shown. MR, AR, AR10, AR2 and } \\
Ark-700 represent the data collected as discussed in the text and as shown \\
in Figures 1a, 1b, 1c, 2 and 3 respectively. Means have been rounded to two \\
decimal places resulting in many of the components being shown as zero. \\
Means are represented in conventional as well as component notation. \\
\hline $\begin{array}{l}\text { Measurement } \\
\text { Set }\end{array}$ & Sph & Cyl & Axis & $\mathbf{I}$ & $\mathbf{J}$ & $\mathbf{K}$ \\
\hline MR (Fig1a) & -5.07 & 0.00 & 179 & -5.07 & 0.00 & 0.00 \\
\hline AR (Fig 1b) & -5.06 & 0.00 & 0 & -5.06 & 0.00 & 0.00 \\
\hline $\begin{array}{l}\text { AR10 (Fig } \\
\text { 1c) }\end{array}$ & -5.05 & 0.00 & 175 & -5.05 & 0.00 & 0.00 \\
\hline AR2 (Fig 2) & -5.03 & 0.00 & 172 & -5.03 & 0.00 & 0.00 \\
\hline $\begin{array}{l}\text { ARK-700 } \\
\text { (Fig 3) }\end{array}$ & -5.06 & 0.07 & 61 & -5.10 & -0.02 & 0.03 \\
\hline
\end{tabular}

Antistigmatic variation is seen in some of the data points showing the presence of antistigmatic components of refractive state. The mean of the measurements presented in Figure 2 can be seen in Table 1. Two possible collections of data points are also evident with one cluster positioned about the origin of the axes and the other positioned slightly more myopically. This presentation could indicate a possible binomial distribution of the data. A MANOVA was conducted on the data discussed to this point. At the $95 \%$ level of confidence all means and variances were shown to be significantly different. A hypothesis test was conducted to determine if the means of the data collected under the same conditions two months apart (data represented in Figures $1 \mathrm{~b}$ and 2) are different. At the 95\% level of confidence the means were shown to be significantly different.

A Nidek ARK-700 autorefractor was used to take 50 measurements of the same test eye so that some comparison could be made in terms of the accuracy of the OPD-scan. The instrument was refocused after each measurement and the user triggered the instrument when it was adequately focused. The measurements taken using the ARK-700 are shown in Figure 3. It is immediately apparent that there is more antistigmatic variation in these measurements compared with the measurements obtained using the OPD-scan, shown by the large clus- 
ter of data points. A single point, positioned below the large cluster, in this orientation, might indicate an outlier. The appearance of the data points in Figure 3 is more in line with the data taken by other members of the OSRG in other investigations. The mean of the 50 measurements is shown in Table 1.

To put the data collected in this investigation into perspective Figure 4a shows all the data collected (using both the OPD-scan as well as the ARK-700) presented on one scatter plot. The axis length is $1 \mathrm{D}$. A tight cluster with some stigmatic variation is seen positioned just below the origin of the axes. Some width to the cluster exists showing the antistigmatic variation present in the data colleted using the ARK-700 autorefractor. Figure 4b shows the same data as Figure 4a with the data collected from the ARK-700 excluded. A slightly elongated cluster is seen showing the stigmatic nature of the variation. There is little waist to the cluster showing the small amounts of antistigmatic variation in the data collected. The two possible outliers mentioned above (seen in Figure 2) can be seen positioned below the primary cluster (in this orientation).

\section{Discussion}

Two hundred measurements of refractive state were obtained from a test eye using a Nidek ARK-10000 OPD-scan. Different methods of focusing and triggering the instrument were used in an attempt to determine how repeatable such measures would be. Although the means of 150 (MR, AR, A10) measurements of refractive state were shown to be significantly different at a $95 \%$ level of confidence the differences were small (see Table 1), the range of the stigmatic component being 0.02 D. Even though different methods were used to trigger and focus the instrument there is little clinical difference between the means. Because the data collected by means of the instrument triggering itself (AR) were purely stigmatic, a finding that has not been seen before, a second set of measurements were obtained using the same method two months later. The means between the two sets of measurements determined two months apart were found to be significantly different at a $95 \%$ level of confidence (AR and AR2). However, clinically, the differences are probably insignificant.

In an attempt to compare the OPD-scan with a conventional autorefractor, 50 measurements were taken of the test eye using a Nidek ARK700 autorefractor. The instrument was refocused after each measurement and the operator decided when to trigger the instrument (in the same way that data was determined for MR). A statistically significant difference exists between these two means (see MR and ARK-700 in Table 1). Nevertheless, the differences between the two means are again probably clinically insignificant.

When all five sets of data are reproduced on one set of axes with an axis length of 1.00 $\mathrm{D}$ a small cluster is evident (see Figure 4a), when the ARK-700 data are removed an even smaller cluster is seen (see Figure $4 b$ ). The size and tightness of the cluster in Figure 4a shows the small amount of variation that occurred in determining 250 measures of refractive state from the same test eye. Relative to the $1 \mathrm{D}$ length of the axes it can be seen that the variation in the measurements is small and probably clinically insignificant. Although the means of the different methods used to trigger and focus the instrument are significantly different at a $95 \%$ level of confidence clinically the method used to focus and trigger the instrument probably makes no difference (see Table 1).

The dioptric power of the test eye is stated as -4.92 by the manufacturer. The OPD- scan and the ARK-700 produce a slightly more myopic measure of the test eye (at most $0.15 \mathrm{D}$ ). The discrepancy between the stated power of the test eye and that determined in this study could be due to various reasons: firstly, the stated power is incorrect and the test eye is in fact more myopic; secondly the instruments may have tended to measure the test eye as slightly more myopic due to some calibration error in the instruments. The likelihood that two different 
instruments measure a test eye as being more myopic by almost the same amount is small.

The results of this study suggest that the OPD-scan provides a repeatable and accurate measure of refractive state of a static test eye. Some variation in the measurements does exist but it is small. Clinically it is probably safe to state that the OPD-scan can be used to determine an accurate and repeatable indication of refractive state of a human eye. However, the clinician needs to remember that the human eye is not static and that proximal accommodation, accommodative spasm and propinquity, among others, can influence the measurement of refractive state when using automated refractometers. Researchers, on the other hand, need to keep the limitations of such instruments in mind when conducting their research.

\section{References}

1. The Nidek-OPD scan. Nidek information pamphlet.

2. Nidek operators manual. 2004 Nidek Co (Ltd).

3. Perrigin DM, Perrigin J, Grosvenor T. A clinical evaluation of the American Optical SR-III subjective refractor. Am J Optom Physiol Opt 198158 581-589.

4. Wesemann W, Rassow B. Automated infrared refractorsa comparative study. Am J Optom Physiol Opt 198764 627-638.

5. Elliot DB, Wilkes RD. A clinical evaluation of the Topcon RM 6000 autorefractor. Clin Exp Optom 1989 72 150-153.

6. Raj PS, Villada JR, Lewis AE, Joyce PW, Watson A. Comparative evaluation of the Allergan-Humphrey 570 and Canon R-1 autorefractors: I objective autorefraction in normal subjects. Eye 19926 284-286.

7. Villada JR, Raj PS, Lewis AE, Joyce PW, Watson A. Comparative evaluation of the Allergan-Humphrey 570 and Canon R-1 autorefractors: II objective autorefraction in psuedophakes. Eye 19926 287-289.

8. Solomons H. Comparison of autorefractors. Optician 1993 April 36-44.

9. McCaghrey GE, Matthews FE. Clinical evaluation of a range of autorefractors. Ophthal Physiol Opt 199313 $129-137$.

10. Grosvenor T, Perrigin DM, Perrigin J. Comparison of American Optical SR-IV refractive data with clinical refractive data on a group of myopic children. Am J Optom Physiol Opt 198360 224-235.

11. Perrigin DM, Grosvenor T, Reis A, Perrigin J. Comparison of Dioptron Nova refractive data with conventional refractive data. Am J Optom Physiol Opt 1984 61 479-483.

12. Berman M, Nelson P, Caden B. Objective refractors: comparison of retinoscopy and automated techniques. Am J Optom Physiol Opt 198461 204-209.

13. Grosvenor T, Perrigin DM, Perrigin J. Three-way comparison of retinoscopy subjective and Dioptron Nova refractive findings. Am J Optom Physiol Opt 198562 43-65.

14. Perrigin DM, Grosvenor T, Perrigin J. Comparison of refractive findings obtained by the Bausch and Lomb IVEX and by conventional clinical refraction. Am J Optom Physiol Opt 198562 562-567.

15. Reeves BC, Hill AR, Carter SC, Sparrow J. Evaluation of two infrared autorefractors in psuedoaphakia. Ophthal Physiol Opt 199212 405-410.

16. Malan DJ. The excess of objective refraction over subjective clinical refraction: methods of analysis and results. Masters thesis, Rand Afrikaans University, Johannesburg, South Africa 1994.

17. Richter SM. Refractive status of children: intra-ocular variation and inter-ocular spread. Masters thesis, Rand Afrikaans University, Johannesburg, South Africa 2000.

18. Du Toit I. The effect of physical exercise on keratometric variation in the human eye. Masters thesis, Rand Afrikaans University, Johannesburg, South Africa 2001.

19. Rubin A. Short-term variation of refractive behavior in human eyes. Masters thesis, Rand Afrikaans University, Johannesburg, South Africa 1993.

20. MacKenzie G, Badenhorst A, Brand C, Van Niekerk F, Burger S, Hall G. Variance in daily autokeratometric means across the menstrual cycle. S Afr Optom 200160 89-96.

21. Rosenfield M, Chiu NN. Repeatability of subjective and objective refraction. Optom Vis Sci 199572 577-579.

22. Bullimore MA, Fusaro RE, Adams CW. The repeatability of automated and clinician refraction. Optom Vis Sci 199875 617-622.

23. Gillan WDH. Time effects on autorefractor measurements. S Afr Optom 200160 85-88.

24. Gillan WDH. Autorefractor focus and refractive variation of a test eye. S Afr Optom 200059 115-119. 